\title{
Evidence of vortex driven primary breakup in high pressure fuel injection
}

\author{
Junmei Shi*1, Pablo Aguado Lopez ${ }^{1}$, Eduardo Gomez Santos ${ }^{1}$, Noureddine \\ Guerrassi ${ }^{1}$, Gavin Dober ${ }^{1}$, Wolfgang Bauer ${ }^{2}$, Ming-Chia Lai ${ }^{3}$, Jin Wang ${ }^{4}$ \\ ${ }^{1}$ Delphi Automotive Systems, Bascharage, Luxembourg \\ ${ }^{2}$ ANSYS Germany GmbH, Otterfing, Germany \\ ${ }^{3}$ Wayne State University, USA \\ 4Argonne National Laboratory, USA
}

*Corresponding author: Junmei.shi@delphi.com

\begin{abstract}
This paper is to present a detailed case study on how the nozzle flow dynamics influences the primary breakup in the spray formation process of diesel injection. The investigation was based on a 3-hole real-application nozzle with highly tapered injection holes using a URANS-LES (Large Eddy Simulation) hybrid approach in combination with the coupled Volume of Fluid (VOF) and Level Set method. High resolution LES was applied to simultaneously resolve the multi-scale nozzle flow dynamics downstream of the needle seat and the primary breakup process in the near-nozzle spray. Phase Contrast X-ray imaging (PCX) was applied to characterize the liquid-gas interfaces in the near-nozzle spray for validation purposes. The results provide detailed information on how the vortex shedding and vortex interactions in the injection hole drives the jet deformation, ligament and droplet formation in the primary breakup process.
\end{abstract}

\section{Keywords}

Primary breakup, Fuel injection, Vortex dynamics, LES, Phase Contrast X-ray imaging

\section{Introduction}

Clean internal combustion engine technology improvement requires the capability to control and optimise the fuelgas mixing, ignition, and combustion process. However, how to transfer the individual engine requirements on the spray to a specific nozzle design still remains a challenging engineering task. One blocking point is the lack of detailed understanding on the fundamental physics of the primary breakup process. This process involves highly complex multi-phase and multi-scale fluid dynamics phenomena, including turbulence, cavitation and their interaction. A significant number of investigations have been dedicated to the cavitation phenomenon over the last 30 years. As for turbulence, the scales and dynamics of the vortex structures in the nozzle flow need to be understood. Two experimental investigations have reported vortex phenomena in injection nozzles. One is the cavitation visualisation of (1) in a real-size VCO nozzle. The vapour distribution in the injection holes indicated the occurrence of strong swirling vortex structures and vortex shedding. Though the investigation was focused on the in-nozzle flow, the authors proposed that the vortex shedding can impact the jet breakup downstream of the injection hole exit. Another is the string cavitation characterization in a scale-up nozzle (2), which demonstrated that string cavitation is caused by large-scale vortex strings in the sac and injection holes and has a correlation with the fluctuation of the spray dispersion angle. Nevertheless, the vortex structures are expected to be much more complex and have richer scales in real applications due to much higher velocity gradients. It is almost impossible to make detailed experimental characterization of field turbulence and vortex dynamics inside a realsize nozzle due to the small dimensions and high speed of the problem. CFD simulation is advantageous over measurement techniques to gain insight into the nozzle flow dynamics and vortex structures and their impact on the spray as shown in (3), (4). In order to resolve the involved multi scale and dynamic phenomena, ScaleResolved Simulation approaches (SRS), such as LES, are needed.

For the primary breakup diagnostic, several effective visualization techniques have been developed in the current century. It is worth mentioning the high resolution PCX imaging developed at Argonne National Lab (5), and the recent application of Transmitted Light Microscopy to the near-nozzle spray visualization (6). Both tools are useful for the characterization of the liquid-gas interface in the primary breakup process having different strengths. From the simulation point of view, interface tracking techniques like the Level-set method have been successfully applied to resolve the liquid-gas interface in the ligament and droplet formation process (7) (8). In order to obtain 
detailed information on how the fluid dynamic instabilities in the nozzle flow trigger ligament and droplet formation and how the nozzle geometry influences those processes and consequently the spray structure, techniques allowing for simultaneous diagnostic of the nozzle flow and the near-nozzle spray are needed. Considering the limitation of measurement techniques for the characterization of field turbulence in a real-size fuel injection nozzle, Scale-Resolved Simulation is a more feasible tool for this purpose. The main issue for simulation is how to deal with the cavitation phenomenon using an interface tracking technique, which naturally requires applying Direct Numerical Simulation (DNS) and is still beyond the capability of most available CFD codes and computational power. An alternative is to treat cavitation by using the Volume of Fluid (VOF) approach, which is a naturally conservative method tracking the volume fraction of a particular phase in each cell rather than the interface itself, being effective for the in-nozzle flow analysis but at the expense of having an excessive numerical diffusion for the jet breakup prediction. This approach might be useful for predicting the liquid jet fragmentation and fuel distribution in the breakup process, but might not be able to provide details for the droplet formation process.

Considering the strength and limitations of both measurement and simulation techniques, the authors have adopted a correlation based approach for years to work out understanding on how nozzle design and operating conditions influence on the spray behaviour. This approach involved the application of simulation for the nozzle flow and measurement techniques for the near-nozzle spray characterization and identifying links between both (4), (9), (10), (11). These successful studies have given the authors confidence in the simulation tools (ANSYS CFX and Fluent) for the nozzle flow diagnostic. In this work we present a detailed case study on the primary breakup of Diesel fuel jet injected from a so-called High Performance atomization (HP) hole nozzle (12). The HP hole uses very high hole taper (Kfactor $=($ Dout-Din $) / 10[\mu \mathrm{m}]=5$, see Figure 1$)$ to increase the hydraulic efficiency and the spray momentum rate. The target is to make a direct investigation on how the nozzle flow dynamic impacts the primary breakup and to reveal the flow dynamic processes in detail. Since the high hole taper prevents the occurrence of cavitation, the coupled VOF-Level Set LES method (13) can be applied simultaneously to resolve the nozzle flow and the liquid jet primary breakup. In order to ensure the numerical quality, the influence of grid resolution on the simulation results has been carefully analysed. Phase Contrast $X$ ray imaging $(P C X)$ was applied to visualize the liquid-gas interface structures for the near-nozzle spray to support the simulation analysis. The experimental investigation was carried out for injection pressures from 400bar to 2000 bar using a spray chamber at atmospheric pressure. The simulation case study was carried out for the injection pressure of 800 bar.

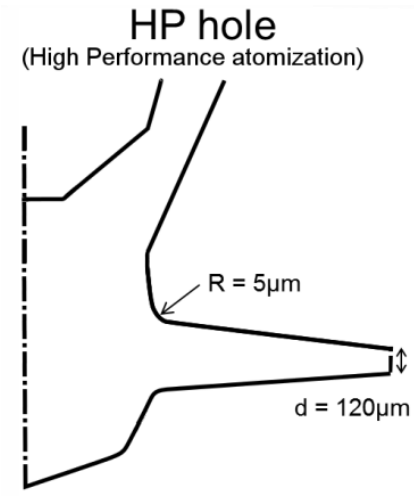

Figure 1. Schematic illustration of injection nozzle geometry

\section{Simulation setup}

The simulation was performed with the software ANSYS Fluent 16.2 adopting a hybrid URANS-LES approach and using a 120-degree sector nozzle model. The computational domain was divided into two subdomains as shown in Figure 2. The sub-domain upstream of the seat sealing was solved with URANS using a tetrahedral mesh and the sub-domain downstream of the seat sealing, including the near-nozzle spray region, with LES using high-quality hexahedral cells. A careful best practice study was carried out to ensure the quality of the simulation results. It was verified that the location of the URANS-LES interface is far enough from the region of interest to avoid causing a distortion of the results. At the URANS-LES interface, only the pressure and velocity fields were interpolated without introducing any artificial disturbance to the LES flow. Regarding the numerical setup, a VOF Level Set method (13) was applied to simultaneously resolve the nozzle flow and jet breakup. A standard k- $\omega$ SST turbulence model was used in the URANS domain and the Wall-Adapting Local Eddy (WALE) sub-grid scale (SGS) viscosity model (14) in the LES domain owing to its ability to correctly predict the near-wall eddy viscosity. 
A fully implicit, 2nd-order time-accurate scheme was used together with a 2nd-order scheme for spatial discretization. In order to ensure sufficient numerical resolution, the following criteria were used: local SGS eddy viscosity ratio below 1 , local CFL number below 2 , and $\mathrm{y}^{+}<1.0$.

Two different meshes were utilized in the discharge volume in order to assess the effects of mesh resolution. A coarser mesh (mesh 1) was defined with $\sim 15$ million cells and an average cell size of $\sim 5.7 \mu \mathrm{m}$, and a finer mesh (mesh 2) with $\sim 31$ million cells and an average cell size of $\sim 3 \mu \mathrm{m}$ for the domain outside of the nozzle. The timestep for mesh 1 was $\Delta t=5 \times 10^{-9} \mathrm{~s}$ and for mesh 2 was $\Delta t=2.5 \times 10^{-9} \mathrm{~s}$ to ensure numerical stability. Each simulation was first run with URANS for $100 \mu$ s and then switched to LES for $50 \mu$ s for initialization purpose to ensure proper development of LES flow. After initialization, the simulation ran further for $100 \mu$ so provide sufficient data for statistical sampling and analysis.

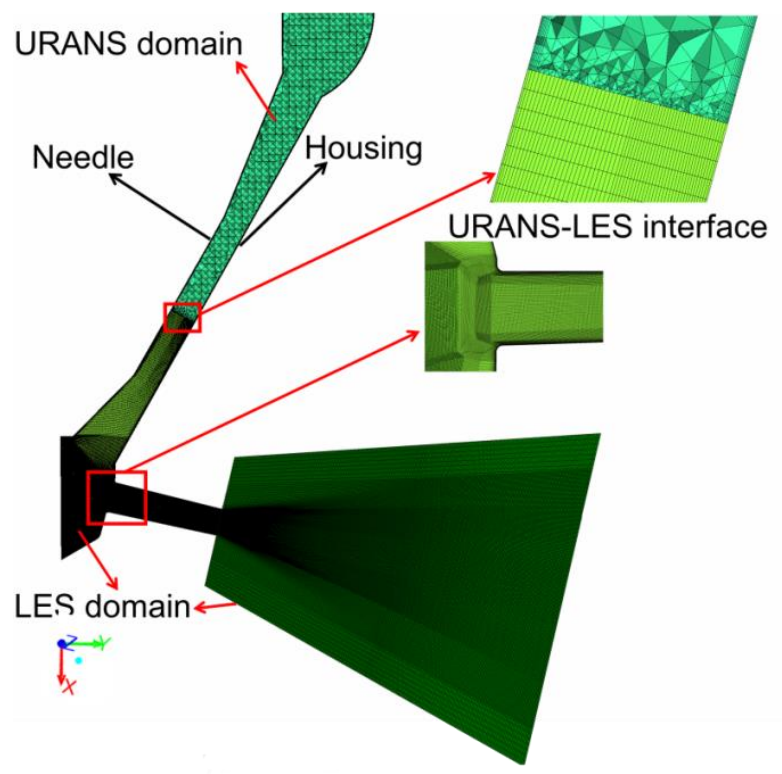

Figure 2: Computational domain decomposition and Hybrid LES mesh for the nozzle tip.

\section{Experimental setup}

The PCX imaging was performed at the XOR 7ID beamline in the Advanced Photon Source (APS) to visualize the near-nozzle spray. The third generation synchrotron $x$-ray beam can produce ultra-short x-ray pulses and weak interaction with the object materials. With these features and the high transmittance of the $x$-ray in dense materials, it becomes possible to capture the instantaneous liquid-gas interface structures in the near-nozzle spray. After passing thought the spray, the x-ray beam forms a phase-contrasted image on a scintillator crystal CCD camera. The field of view of the camera was $1.734 \mathrm{~mm} \times 1.310 \mathrm{~mm}$ with a pixel resolution of $0.66 \mu \mathrm{m} / \mathrm{pixel}$ when a 20 times objective lens was used. The imaging frequency is $50 \mathrm{kHz}$, or $20 \mu \mathrm{s}$ per image. A detailed description of the experimental setup can be found in (9).

\section{Results and discussion}

\section{Liquid-gas interface structures: mesh resolution effect}

Similar liquid core interface structures and jet breakup patterns were predicted on both meshes, but the higher resolution of mesh 2 captured much more small droplets. Ideally, a proper post-processing tool for scale separation and calculation should be developed and used to assess the minimum droplet size which can be captured by each mesh. As this tool was not available a concept of interface diffusion thickness is used instead. This value is calculated using the Level Set function $(\varphi)$ and liquid volume fraction, and is introduced to help estimate the mesh resolution effect on the diffusion of the liquid-gas interface structures. The interface diffusion is a result of mesh resolution and diffusion caused by numerical schemes. Therefore, this method is also useful for a coarse estimation of the smallest droplet resolution as will be explained. The process of the interface smearing due to numerical diffusion is schematically plotted in Figure 3 a) for a single droplet. The Level set function is exactly zero at the interface and has a value equal to the distance to the interface (with a positive or negative sign 
according to the convention for each phase) for any other points (13). Initially, the droplet is bounded by a sharp interface $\varphi=0$ where the liquid volume fraction jumps from $\alpha_{l}=0$ outside the droplet to $\alpha_{l}=1$ inside the droplet. After a number of time steps the interface smears, leading to a smooth volume fraction variation across the interface. In this new state, the region where $\alpha_{l}=1$ is restricted to some cells in the center of the droplet, bounded by a surface where $\varphi=0$. Taking $\alpha_{l}=0.01$ as the threshold of the interface diffusion, the thickness of the diffused interface $d_{1 \%}$ can be estimated using the distance between the volume fraction iso-surface, $\alpha_{l}=0.01$ and the iso-surface $\varphi=0$. Under a symmetric interface diffusion assumption the diameter $d$ of a spherical droplet is $2 r \leq d \leq 2 r+2 d_{1 \%}$, where $r$ is the distance from the droplet core center to the undiffused droplet core interface $\varphi=0$. The smallest spherical droplets near the intact liquid core $\left(\alpha_{l}=1\right)$ are only distributed over one or a few cells, leading to $r \sim 0$. In such cases, $d \sim 2 d_{1 \%}$ is a reasonable estimate of the actual characteristic droplet size.

An instantaneous near-nozzle spray visualisation is presented in Figure 3 b) for both meshes using the instantaneous liquid volume fraction iso-surfaces 0.01 colored by $d_{1 \%}$. The colour scale threshold chosen here is aimed at separating the smallest droplets $d \sim 2 d_{1 \%}<10 \mu \mathrm{m}$ from the larger droplets and the liquid core interface. It is observed that Mesh 1 only captured a few droplets with $d \sim 2 d_{1 \%}<10 \mu \mathrm{m}$ very close to the liquid core during initiation of breakup. Further downstream, only larger droplets and ligaments are resolved with increasing diffusion due to mesh coarsening. In contrast, mesh 2 is fine enough to capture droplets smaller than $10 \mu m$ over the entire primary breakup region modelled. In addition, it is noted that the diffusion thickness is low for the liquid core obtained based on both meshes. Therefore, it is safe to say that the mesh resolution is unlikely to influence the numerical observations of the liquid jet deformation and ligament formation phenomena in the primary breakup process.

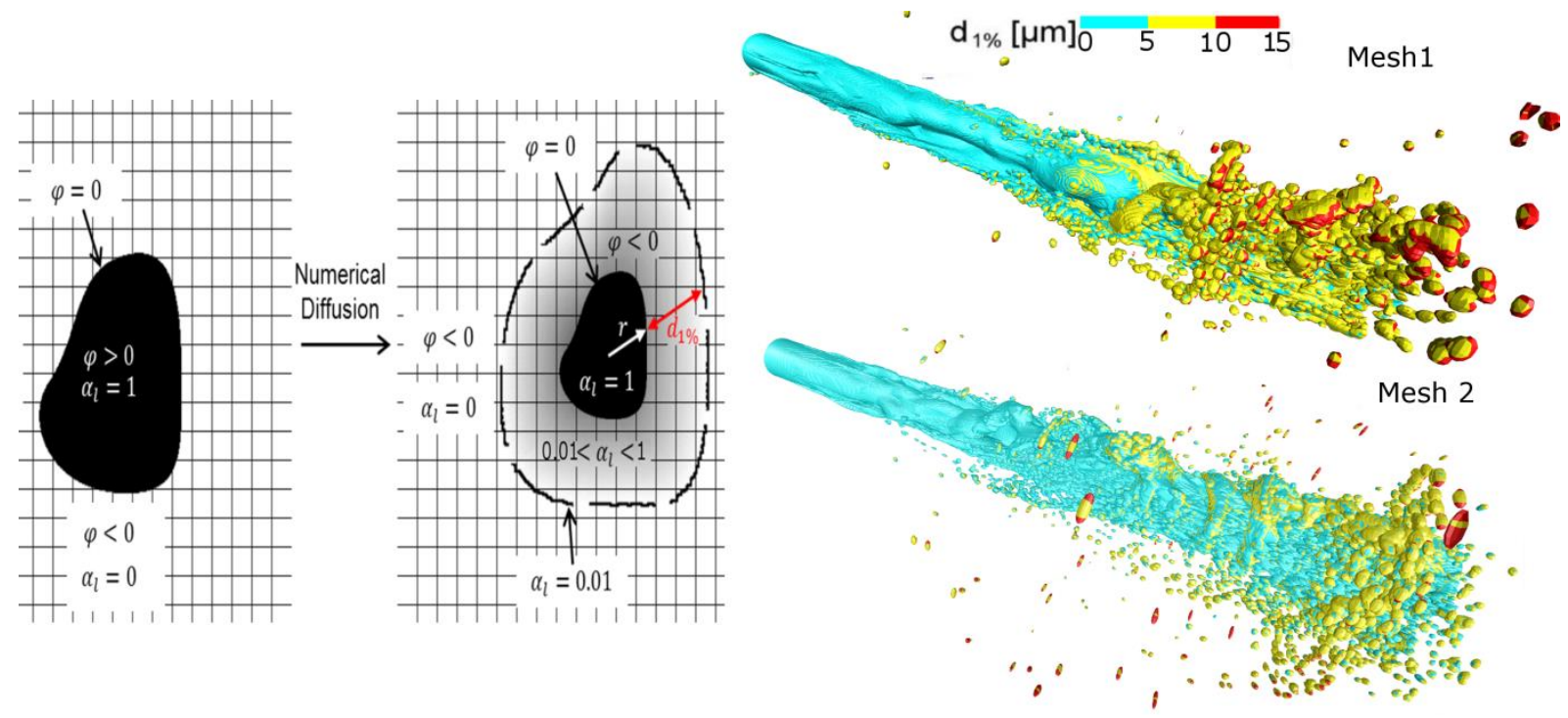

a) Sketch of interface smearing due to numerical diffusion. Sharp interface (left) and diffused interface right.

b) Estimation of droplet size based on $d_{1 \%}$. Liquid volume fraction iso-surface 0.01 colored by $d_{1 \%}$.

Figure 3: Assessment on mesh resolution effect on small droplets

\section{Liquid-gas interface structures: LES solution vs. PCX spray images}

Comparison for the liquid-gas interface structures between LES solution and PCX spray images should be based on a statistical approach. This is hindered by the differences in the physical time durations and the time resolution between simulation and measurement. Spray imaging was performed for an injection duration of $1 \mathrm{~ms}$ at full needle lift and at a time interval of $20 \mu$ s per image (50 images in total). The physical time in simulation was $100 \mu \mathrm{s}$ for both meshes. Flow visualisation images were stored every $5 \times 10^{-8} \mathrm{~s}$. Obviously, the time resolution and the image number in the measurements were not sufficient in the sense of a rigorous statistical analysis of the nearnozzle spray structures, while the physical time duration in the simulation is too short as it is limited by the available computational resources. Under these limitations, effort was made to identify similarities between the instantaneous spray morphologies captured by PCX imaging and by the simulations. 
The LES results for mesh 1 and mesh 2 are treated as independent time series, considering the fact that the initialization has an influence on the flow development. The mesh 1 solution of the near nozzle spray was found to have a close correlation with 7 out of the 50 PCX spray images recorded over the open needle operation time interval. Sample results are presented in Figure 4, where the LES near-nozzle sprays are represented by using the iso-surface of 0.1 liquid volume fraction. The predicted undisturbed liquid core before the initiation of jet breakup is obviously longer than the measurement, but the simulation is able to capture some features observed in the PCX spray images. For example, a linear streak crossing the liquid core very close to the nozzle exit can be recognized from both the predicted and measured spray (Figure 4, left). In particular, a close similarity between both is observed at the lower side of the spray, where the wavy structures begin to break up into smaller structures and droplets. Figure 4 right shows another example. The wavy structures with higher local breakup intensity on the upper side of the spray as recorded by the PCX imaging are also captured in the simulation. In addition, a "horizontal boundary" between the continuous un-atomized liquid core (lower part of the spray) and the upper spray regions with small structures can be well noted both from the PCX spray image and the LES solution.

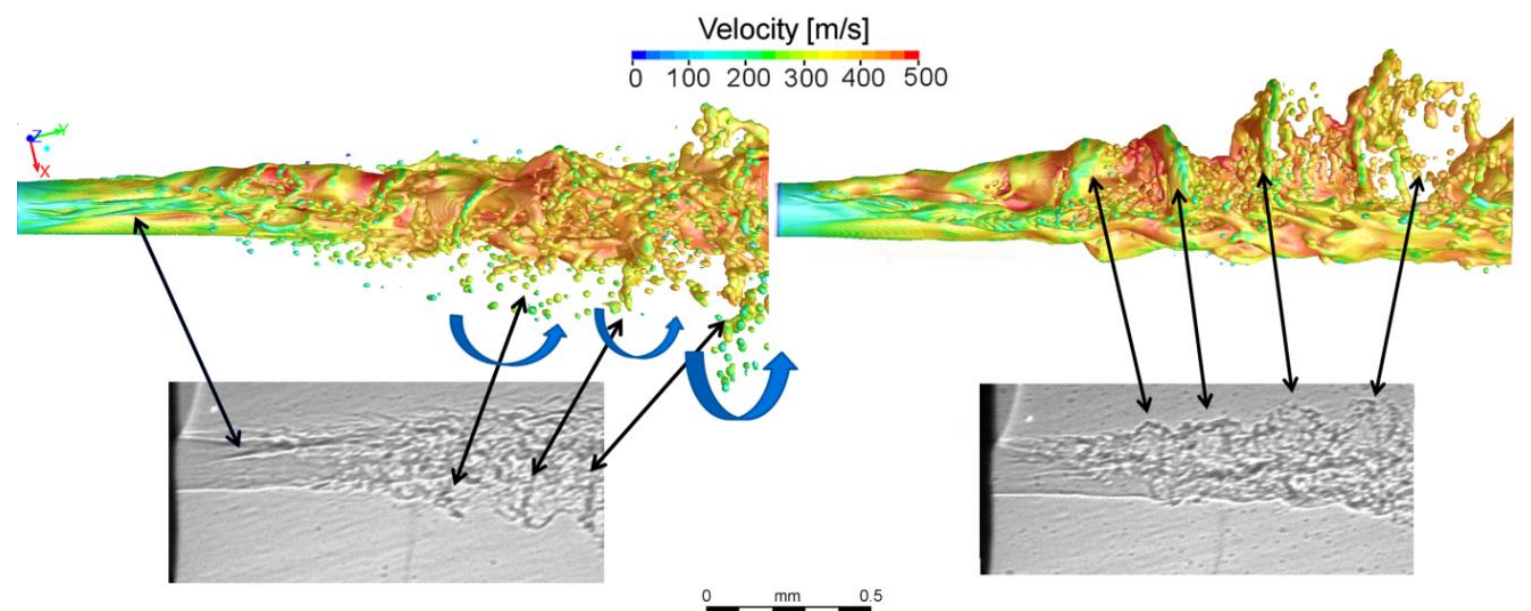

Figure 4: Instantaneous LES liquid volume fraction iso-surfaces (value=0.1) on mesh 1 (top) vs. PCX images (bottom)

The LES solution on mesh 2 was found to produce similar spray features with 8 out of the 50 PCX images. Figure 5 shows for the correlations between some example mesh 2 results and PCX spray images. The LES spray in the left image shows a braid-like (helical) structure appearing on the upper side of the very initial jet, which can also be noticed from the PCX spray image. This type of structures are caused by vortex shedding and rotating string vortices occurring in the nozzle flow and will be discussed in detail in the next section. In addition, similar vertical streaks are observed both in simulation and measurement. The LES spray on the right shows a close similarity in terms of breakup patterns and spray shape to the corresponding mesh 1 result shown on the right of Figure 4, As with the mesh 1 result, the wavy structures on the upper side of the spray correlate well with the PCX image.

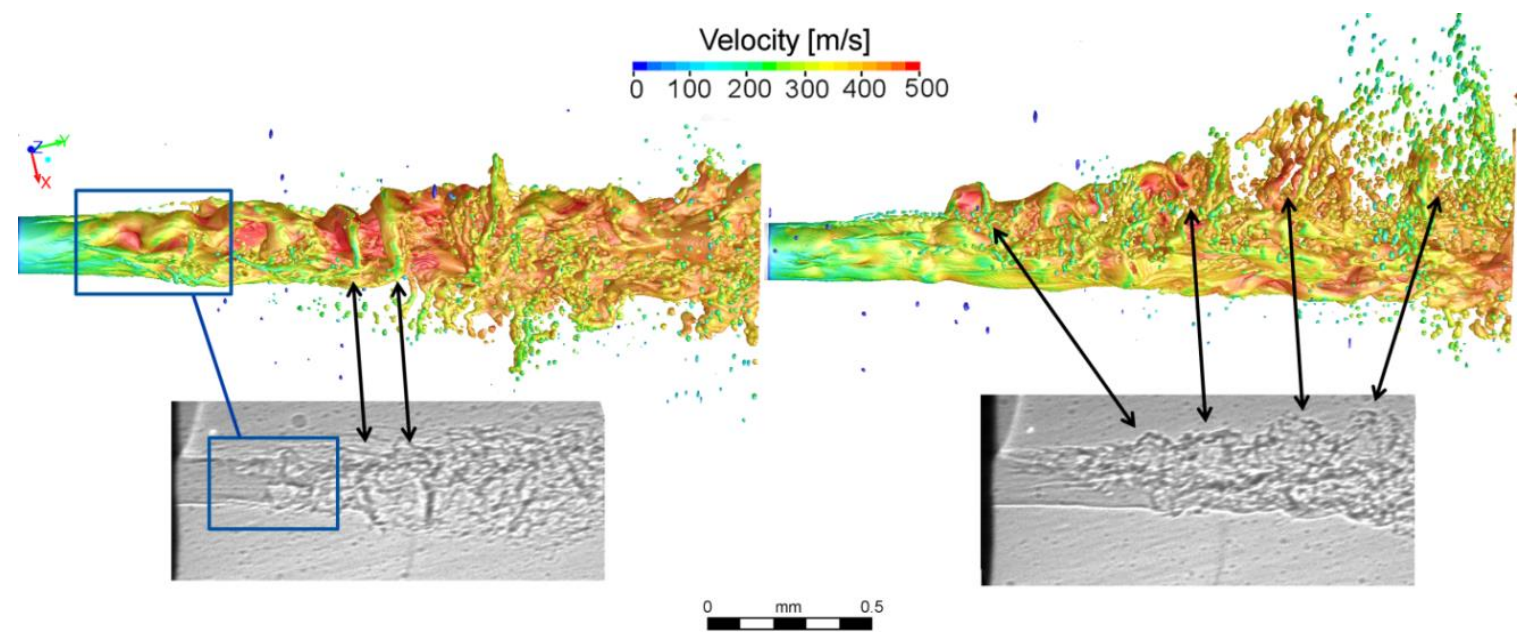

Figure 5: Instantaneous LES liquid volume fraction iso-surfaces (value=0.1) for mesh 2 (top), PCX images (bottom). 
These results show a clear impact of the mesh resolution on the small structures and droplets in the near-nozzle spray. However, both meshes have captured some breakup patterns and morphological features of the spray, which can be recognized in PCX spray images, are therefore valid. Since the mesh 2 results were only very recently obtained in this work, the understanding on the physics of the primary breakup process reported below is mainly derived from the mesh 1 solution.

\section{Vortex driven primary breakup process}

Figure 6 illustrates the correlation between the predicted vortex structures and near-nozzle spray structures obtained on mesh 1 together with a similar PCX spray image. Two type of vortices can be observed in the nozzle flow. Small-scale vortices and vortex shedding occur at the upper lip of injection hole inlet as the flow turns into the hole. At the same time, large-scale string vortices are generated in the bulk flow of weak shear due to flow recirculation in the sac and flow acceleration into the hole. The results indicate that the upper-lip vortex shedding and the interaction between the string vortices and the shed vortices in the nozzle are the triggering mechanism of ligament formation in the primary breakup region. A vortex shedding event produces low momentum vortices and a pulsation in the local flow. The shed vortices interact with the string vortices in the injection hole. As they exit the injection hole they transfer their local instability and their pulsating momentum into the liquid jet, causing its deformation and the development of ligaments.

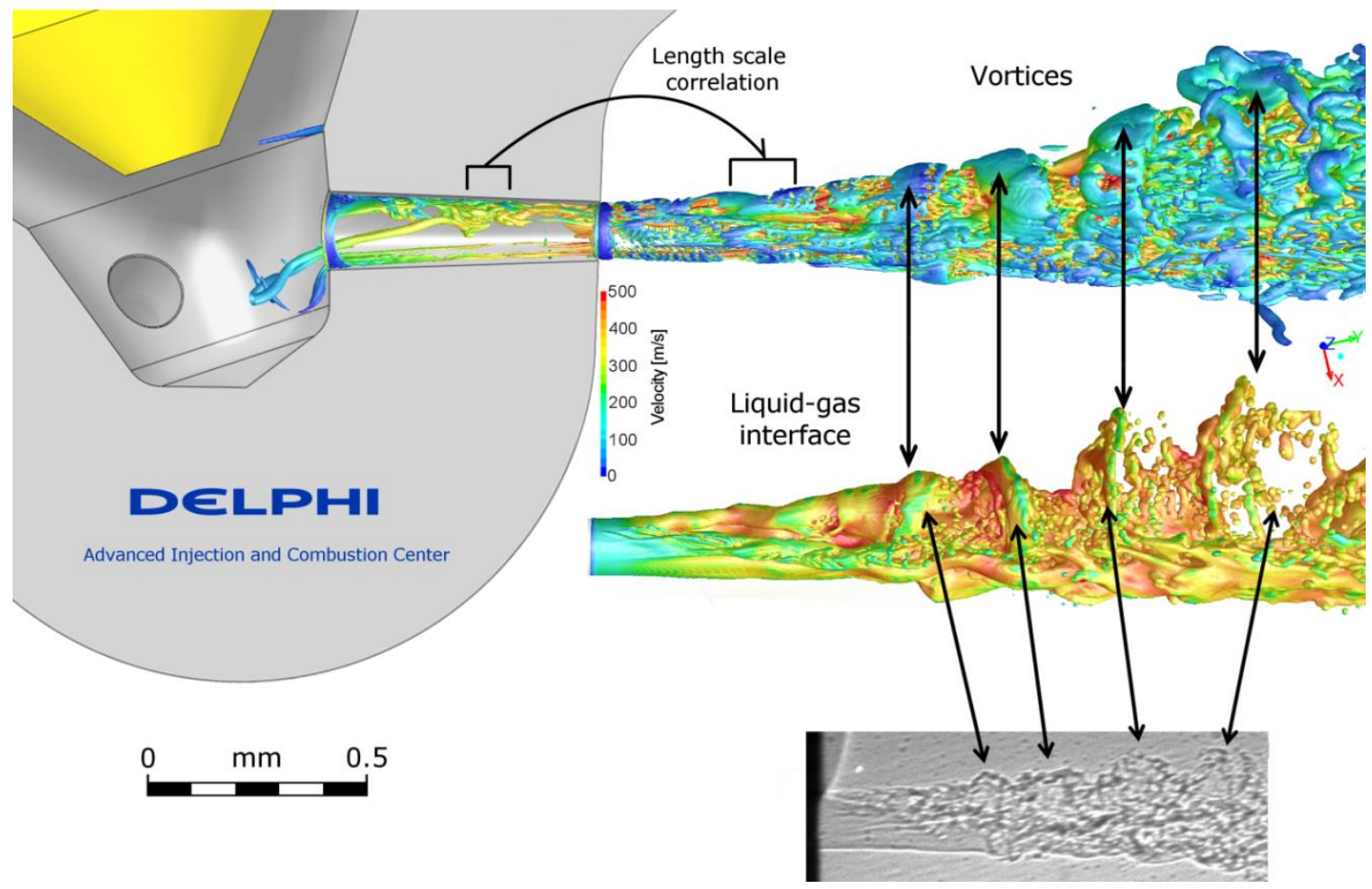

Figure 6: Correlation between vortex shedding in the nozzle and jet breakup: vortex structure $\left(Q=1 \mathrm{e} 13\left[\mathrm{~s}^{-2}\right]\right)$, predicted spray morphology(liquid volume fraction $90 \%$ ), and PCX image (bottom).

This vortex-driven ligament formation and breakup process is illustrated in Figure 7 using a time sequence of instantaneous results for the vortex flow and near-nozzle spray. At a certain time instant $t_{0}$, the string vortices move upwards and interact with the shed vortices close to the hole exit, creating a local flow instability and upward momentum. This pulsating momentum is transported into the near-nozzle flow after a shed vortex leaves the injection hole exit. At $t_{0}+0.45 \mu \mathrm{s}$ the upward moving shed vortex triggers the wavy surface vortex enhanced by the interaction with the surrounding gas. This vortex causes deformation of the liquid jet on the upper side, leading to wavy liquid-gas interface structures. At $t_{0}+2 \mu \mathrm{s}$ the surface vortex gets further developed due to airliquid interaction and the liquid surface deformation continues to grow causing the roll-up of the liquid-gas interface and ligament formation. At $t_{0}+5 \mu \mathrm{s}$ the surface vortices lose their momentum, get separated from the high speed bulk flow and decompose into smaller vortices causing atomization of ligaments and formation of droplets. A detailed video showing this process is available in (15). 


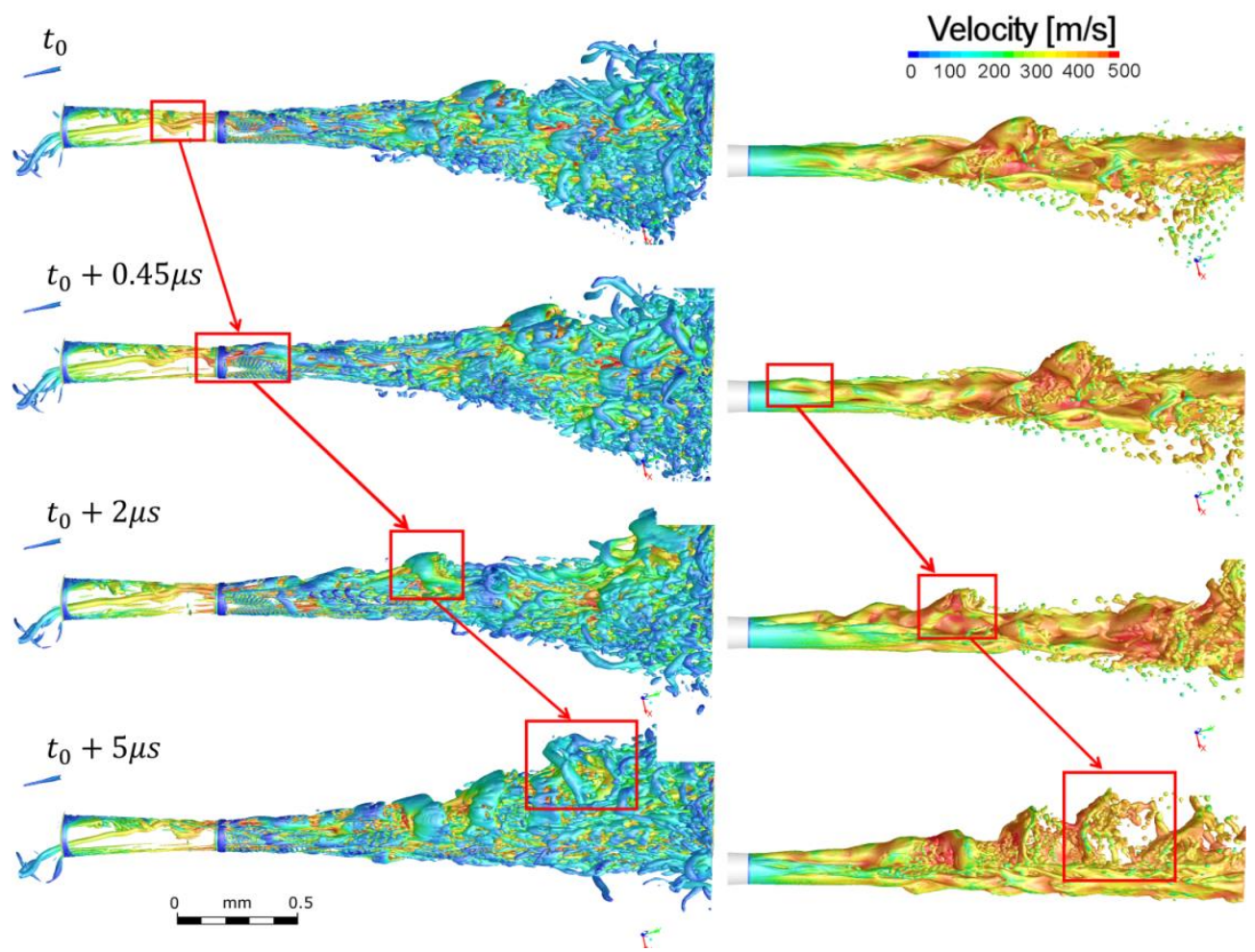

Figure 7: The process of vortex driven ligament formation and jet breakup. Vortex structure $Q=1 \mathrm{e} 13\left[\mathrm{~s}^{-2}\right]$ (left), Liquid-gas interface of the spray using liquid volume fraction (right).

In addition, large scale vortex strings are high energy containing structures. Their morphology, location and motion direction have an important impact on the primary breakup behaviour as is shown in Figure 8 using selected instantaneous results. In case (a), the string vortices move upwards. This triggers liquid core deformation and ligament formation further downstream on the upper side of the jet. The string vortices are pushed downwards by the strong shed vortices at the hole exit in case (b), leading to liquid-core deformation on the lower side. In case (c), the string vortices show an unstable ' $\mathrm{S}$ '-shape motion, causing an earlier jet breakup both on the upper and lower sides. In contrast, relatively undisturbed flow with all large vortices well aligned with the injection hole axis is predicted close to the injection hole exit in (d). Under this situation, weak perturbations on the liquid jet and thus weak jet breakup is observed.
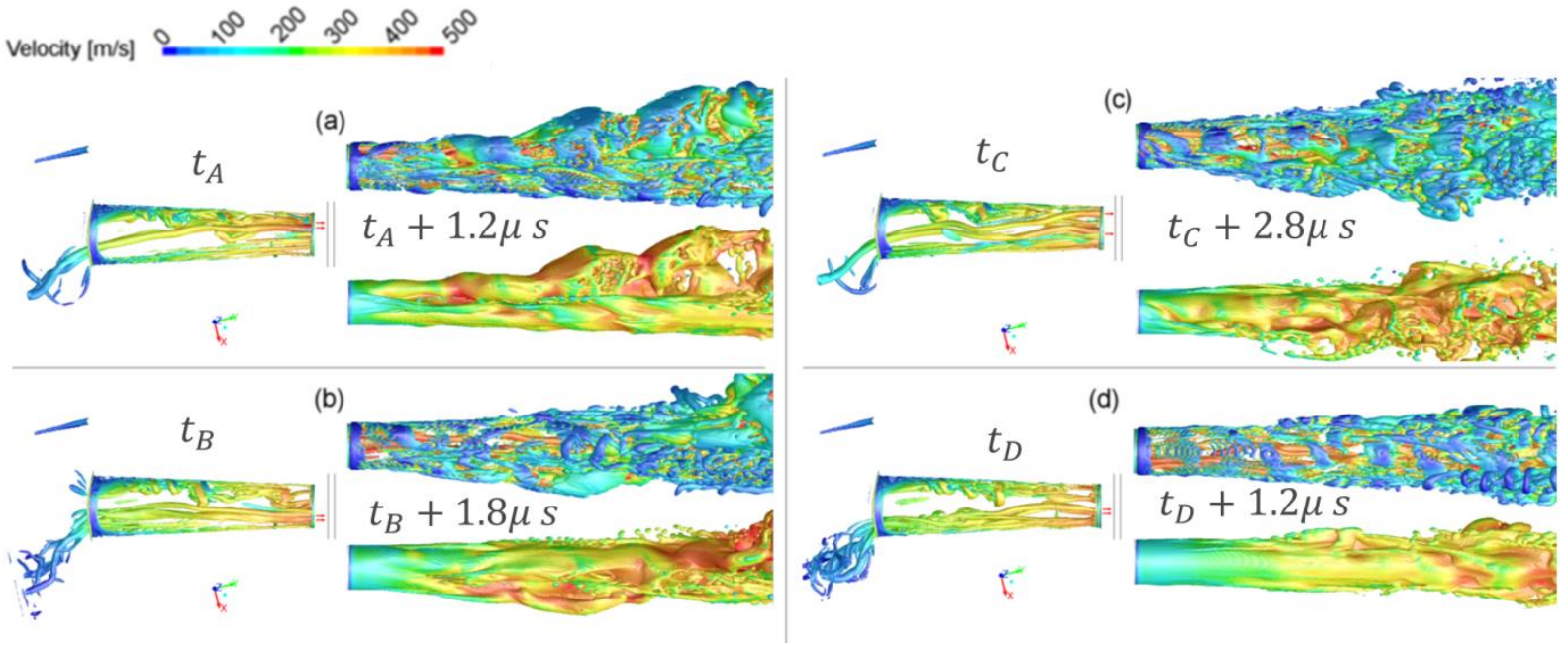

Figure 8: Link between vortex dynamics and liquid-gas interface for different instants. For each case: Vortex structures $\mathrm{Q}=1 \mathrm{e} 13\left[\mathrm{~s}^{-2}\right]$ (left and top-right), Liquid-gas interface of the spray using liquid volume fraction 0.1 (right). Red arrows at the outlet denote the flow direction 


\section{Conclusions}

A detailed case study was carried out on the primary breakup process in high pressure fuel injection based on a production diesel nozzle design. A coupled VOF-Level Set LES simulation methodology was applied to simultaneously resolve the multi-scale flow dynamics in the nozzle and the jet primary breakup process after the hole exit. Phase Contrast X-ray imaging was applied to characterize the liquid-gas interface in the near-nozzle spray. The simulation successfully reproduced many structures of the spray captured by the PCX imaging. It was observed from the simulations that, as the flow is deflected into the nozzle hole, it triggers vortex shedding events, producing high speed, energetic vortex structures and local flow instabilities. These structures continue to develop into the liquid jet and initiate the deformation and ligament formation processes within the primary spray breakup. Additionally, vortex ejection from the nozzle causes small surface vortices at the liquid-gas interface that interact with the surrounding gas and ultimately lead to droplet formation. These results provide evidence for a vortex driven atomization mechanism. With this understanding, fuel injector nozzle designs can be optimized by control and optimization of the vortices.

\section{Acknowledgements}

The FNR funding to the PhD project of Pablo Aguado Lopez (number 588638), the European Union Horizon-2020 Research and Innovation Programme funding to Eduardo Gomez Santos (No 675676), the ANSYS research license grant and the CPU hours grant from Gompute to this work are gratefully acknowledged. The $x$-ray investigation was carried out using the Advanced Photon Source of the Argonne National Laboratory of US Department of Energy. The kind assistance of the scientist of beam line \#32, Dr. Kamel Fezzaa, is highly appreciated by the authors.

\section{References}

1. Cavitation in an asymmetric transparent real-size VCO nozzle. Chaves, Humberto and Schuhbauer, Ingo. 2006. Spray'06, Workshop uber Sprays, Erfassung von Spruhvorgangen und Techniken der Fluidzerstaeubung. 2. Vortex flow and cavitation in diesel injector nozzles. Andriotis A, Gavaises M and Arcoumanis C. 2008, J Fluid Mech, Vol. 10, pp. 195-215.

3. URANS and SAS analysis of flow dynamics in a GDI nozzle. Shi, J.-M.; Wenzlawski, K.; Helie J; Nuglisch, H.; Cousin, J. Brno : s.n., 2010. ILASS-Europe.

4. Using LES and $x$-ray imaging to understand the influence of injection hole geometry on Diesel spray formation. Shi, J.; Aguado Lopez, P.; Dober, G.; Guerrassi, N.; Bauer, B.; Lai, M.-C. Valencia : THIESEL 2016 Conference on Thermo-and Fluid Dynamic Processes in Direct Injection Engines, 2016.

5. X-ray vision of fuel sprays. Wang, Jin. 2, 2005, J Synchrotron Rad., Vol. 12, pp. 197-207.

6. Transmitted light microscopy for visualizing the turbulent primary breakup of a microscale liquid jet. Reddemann, Manuel A. ; Mathieu, Florian ; Kneer, Reinhold. 11, 2013, Experiments of Fluids, Vol. 54, pp. 1-10.

7. On simulating Primary Atomization Using the Refined Level set grid Method. Hermann M. 4, 2011, Atomisation and Sprays, Vol. 21, pp. 283-301.

8. Simulation of liquid jet primary breakup: Dynamics of ligament and droplet formation. Shinjo J. 7, 2010, International Journal of Multiphase Flow, Vol. 36, pp. 513-532.

9. Correlating the Nozzle Flow to Spray and Primary Breakup using Visualization and Multi-phase Simulation. Lai M-C; Wang F; Xie X; Shi J; Dober G; Guerrassi N; Meslem Y. Rouen : s.n., 2014. SIA.

10. Fuel property and fuel temperature effects on internal nozzle flow, atomization and cyclic spray fluctuations of a direct injection spark ignition-injector. Zigan, L, et al., et al. 6, 2013, International Journal of Engine Research, Vol. 14, pp. 543-556.

11. Characterization of Internal flow and Spray of Multihole DI Gasoline Spray using X-ray Imaging and CFD. Ming-Chia Lai, Yi Zheng, Mark Shost, Xingbin Xie, Atsushi Matsumoto, Jin Wang, Xusheng Zhang, Seoksu Moon, Jian Gao, Kamel Fezzaa, Lars Zigan, Ingo Schmitz, Michael Wensing, Alfred Leipertz. 2011. JSAE. Vols. JSAE 20119225, SAE 2011-01-1881.

12. Guerrassi, N.; Doradoux, L.; Garsi, C.; Lesieur, C. Injection nozzle. US 20110215177 A1. 2011.

13. A coupled level set and volume-of-fluid method for computing $3 d$ and axisymmetric incompressible two-phase flows. Sussman, M.; Puckett, E. G. 2, 2000, J. Computational Physics, Vol. 162, pp. 301-337.

14. Subgrid-scale stress modelling based on the square of the velocity gradient tensor flow. Nicoud, F.; Ducros., F. 3, 1999, Turbulence and combustion, Vol. 62, pp. 183-200.

\begin{tabular}{l} 
15. Delphi Automotive Systems Luxembourg. ANSYS Hall of FAME 2017. [Online] \\
\hline
\end{tabular} http://www.ansys.com/other/hall-of-fame. 